\title{
Geburtstag in der Pollensaison
}

\author{
Infektionen der oberen Atemwege können Wegbereiter einer \\ allergischen Sensibilisierung sein. Allerdings sind nicht alle Kinder \\ gleichermaßen betroffen. In einer aktuellen Studie wurde die Pollen- \\ exposition bei der Geburt als Co-Faktor untersucht.
}

$\mathrm{n}$ prospektiven Kohortenstudien wird weltweit nach für die Entwicklung einer Atopie prädisponierenden Umweltfaktoren gesucht. Infektionen der oberen Atemwege erwiesen sich in manchen - aber nicht allen - Studien als ein Risikofaktor. Wichtig ist möglicherweise der genaue Zeitpunkt oder die Gesamtzahl der frühkindlichen Infekte.

Im Rahmen einer prospektiven Kohortenstudie wurde auf der Insel Tasmanien der Einfluss von Infektionen der oberen Atemwege während der ersten Lebensmonate und der Geburtsjahreszeit auf die spätere Entwicklung von Allergien geprüft. Die Forscher konzentrierten sich dabei auf die Rhinitis allergica und eine Sensibilisierung gegen Weidelgras (Lolium perenne). Die Gräsersaison in Tasmanien liegt in den Monaten November und Dezember.

Ausgewertet wurden die Daten von Kindern, die 1988 oder 1989 geboren worden waren und acht Jahre $(\mathrm{n}=498$, erste Gruppe) bzw. 16 Jahre $(\mathrm{n}=415$, zweite Gruppe) lang beobachtet wurden. Infektionen der oberen Atemwege doku-

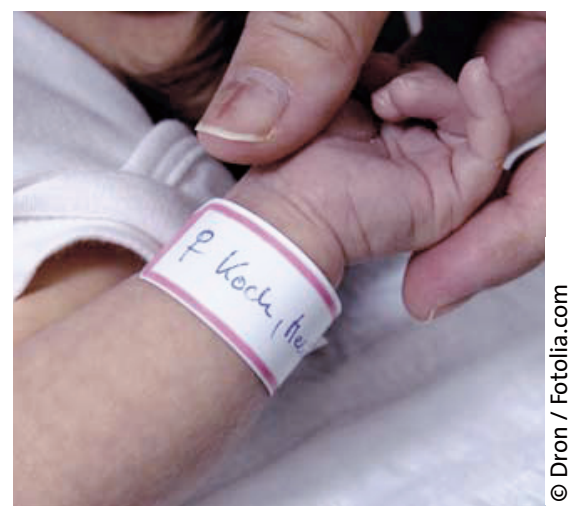

Der Name von den Eltern, das Rhinitisrisiko von der Jahreszeit?

mentierten die Eltern. Der Nachweis der Sensibilisierung erfolgte im Alter von acht Jahren durch einen Hauttest bzw. im Alter von 16 Jahren durch den Nachweis von spezifischem IgE. Das Vorliegen einer Rhinitis wurde mit einem Fragebogen evaluiert.

In der ersten Gruppe hatten 44 $(9,6 \%)$ und in der zweiten Gruppe 47 $(12,5 \%)$ während der Pollensaison Ge- burtstag. Bei Kindern der ersten Gruppe, die während der Pollensaison geboren worden waren, war ein frühkindlicher Infekt der oberen Atemwege signifikant mit einer Sensibilisierung gegen Gräser assoziiert (OR 5,80) - nicht aber bei Kindern, die außerhalb der Pollensaison Geburtstag hatten (OR 0,62). Entsprechend bestand auch ein signifikanter $\mathrm{Zu}$ sammenhang zwischen frühkindlichen Infekten und Heuschnupfen - der wiederum nur bei den Kindern bestand, die während der Gräsersaison geboren worden waren. Die Assoziation zwischen frühkindlichen Atemwegsinfekten und vermehrter Sensibilisierung bei Geburt in der Pollenflugzeit war allerdings nur bei den achtjährigen Kindern, nicht mehr bei den Jugendlichen der zweiten Studiengruppe nachweisbar.

Fazit: Sensibilisierungen gegen Gräser und Heuschnupfen treten signifikant häufiger auf, wenn es zu frühen Infekten der Atemwege kam. Dieser Zusammenhang gilt nach den Daten dieser Studie aber nur für Kinder, die während der Gräserpollensaison geboren wurden. $b k$

Kemp A et al. The interaction between early life upper respiratory tract infection and birth during the pollen season on rye-sensitized hay fever and ryegrass sensitization - a birth cohort study. Pediatr Allergy Immunol 2009; 20: 536-44

\section{Paracetamol und Asthma - ein Update}

\section{Mehrere epidemiologische Studien hatten bereits auf ein erhöhtes Asthmarisiko nach Anwendung von Paracetamol hingewiesen. Die Ergebnisse waren aber insgesamt widersprüchlich. Eine große Meta- analyse sollte jetzt Klarheit bringen.}

K anadische Forscher versuchten, in einer großen Metaanalyse die Beziehung zwischen der Einnahme von Paracetamol und dem Asthmarisiko bei Kindern und Erwachsenen zu quantifizieren. Sie durchsuchten dazu alle großen medizinischen Literaturdatenbanken wie Medline (1966-2008) und Embase (1980-2009) nach einschlägigen Studien. Die Qualität der Studien wur- de von zwei Gutachtern eingeschätzt. Die Metaanalyse umfasste insgesamt 19 klinische Studien - 13 Querschnitts-, vier Kohorten- und zwei Fall-KontrollStudien - mit insgesamt 425.140 Teilnehmern.

Das Asthmarisiko war bei Einnahme von Paracetamol um den Faktor 1,63 erhöht (95\%-Konfidenzintervall [95\%-KI] 1,46-1,77). Nahm eine Frau während der Schwangerschaft Paracetamol ein, erhöhte dies das spätere Asthmarisiko des ungeborenen Kindes auf 1,28 (95\%-KI 1,16-4,1).

Fazit: Nach dieser Metaanalyse erhöht Paracetamol das Asthmarisiko bei Kindern und Erwachsenen, wobei aber kein kausaler Zusammenhang bewiesen ist. Dies sollten prospektive, randomisierte Studien klären. Auch die Diagnose Asthma ist klinisch zu sichern, in den vorliegenden Studien wurde oft nur eine Selbsteinschätzung vorgenommen. $\mathrm{km}$

Etminan $\mathbf{M}$ et al. Acetaminophen use and the risk of asthma in children and adults. A systematic review and metaanalysis.

Chest 2009; 236: 1202-10 